\title{
Artificial intelligence in education and assessment methods
}

\author{
Balqis Al Braiki, Saad Harous, Nazar Zaki, Fady Alnajjar \\ College of Information Technology, United Arab Enirates University, UAE
}

\begin{tabular}{l} 
Article Info \\
\hline Article history: \\
Received Oct 9, 2019 \\
Revised Dec 11, 2019 \\
Accepted Feb 4, 2020 \\
\hline Keywords: \\
Artificial intelligence \\
Course-assessment \\
Education \\
Machine learning
\end{tabular}

Machine learning

\begin{abstract}
Today, artificial intelligence has proliferated to reach almost every wing of daily life, perhaps one of the most sensitive of these being education. While teaching, insofar as it involves training human minds, is still mostly a form of art rather than a regular science, the taking up of this elitist job by computers has triggered much debate and controversy, involving the teaching community as much as the select corporate AI giants who strive to create computers capable of teaching better than humans. This paper surveys the most relevant studies carried out in this field to date. First, it introduces $\mathrm{AI}$ and describes the different AI applications in field of education and course assessment. It then goes on to list the most common topics in the educational context that have been resolved through $\mathrm{AI}$ and machine learning techniques, and finally, some of the most promising future lines of research are discussed.
\end{abstract}

This is an open access article under the $\underline{C C B Y-S A}$ license.

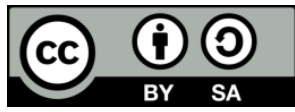

\section{Corresponding Author:}

Saad Harous,

Department of Computer Science and Software Engineering,

College of Information technology,

United Arab Emirates University,

Al Ain 15551, UAE.

Email: harous@uaeu.ac.ae

\section{INTRODUCTION}

Artificial intelligence is the replication of human proficiency processes such as language translation, speech, visual recognition, and virtual decision making by robots and machines. According to Oxford dictionary, it defines artificial intelligence (AI) as the theory and development of computer systems, which are able to perform tasks normally requiring human intelligence. Artificial intelligence (AI) is a branch of computer engineering, designed to create machines that behave like humans [1]. To create machines with the capacity to conduct thought processing and produce behavioral responses like human beings requires a considerable degree of advanced innovation. Artificial intelligence can now derive new methods of teaching, education and learning, and it may alter the social considerations in ways that indicate new challenges for educational affiliations. It may be responsible for the amplification of skill differences or polarization of jobs, or it may become an option for balancing opportunities for knowledge and growth.

From the year 2013, when Frey and Osborne stated that approximately half of US jobs were at significant risk of becoming programmed, artificial intelligence has been one of the most important considerations for policymakers [2]. Several researches have replicated and modified the findings provided in this study, and the general agreement now is that artificial intelligence will drive important changes in the labor market. Multiple characteristics and emotional intelligence traits that were critical in the past are becoming automated, and several professions and jobs will become outdated or changed with the consistent use of artificial intelligence. Furthermore, there have been increasing demands for individuals trained in artificial intelligence development, resulting in hefty salaries and sign-up charges. China, already 
one of the most advanced countries techno- logically, aims to lead the world in artificial intelligence by growing an ecosystem of robots and programmed materials worth 150 billion USD by 2030 .

The US Department of Defense invested approximately 2.5 billion USD in artificial intelligence initiatives for the year 2017, and private investments overall have possibly exceeded 20 billion USD per year. In 2018, there were more than 1200 artificial start-ups in Europe [3], and the European Commission aims to increase public and private associations in the EU artificial intelligence domains by at least 20 billion Euros by the end of year 2020. For particular functions, artificial intelligence already surpasses human capabilities. In 2018, having completed only about one month of system development, academics at Stanford were able to use AI to highlight 14 different teaching methods which could be utilized for different subjects and topics [4]. In May 2018, Google CEO exhibited his AI system. Duplex programming was capable of autonomously scheduling telephone appointments, while making people think they were talking to another human being. While all of its potential, AI is not without critics. One challenge to the dominant learning models applied in artificial intelligence is that they perceive the world as a repetition of historic events. Most of the functional categories and notions of success that are implemented in their preparation are supported by humans.

When a machine (which typically refers to a computer), or more generically, a device, imitates the cognitive functions associated with the human mind (such as solving a problem or learning something in order to effectively understand and simulate human speech or handwriting, play games (like chess or Go), drive cars, and find patterns in complex data such as images or videos) we call this human-like faculty artificial intelligence (AI) [5]. In the 1950s, considered the birth period of AI, Turing tried to define when a system designed by humans could be called intelligent. He proposed the idea of a human trying to distinguish between a conversation with man and one with a machine; if the distinction was not detectable, it iss an intelligent system, or a system with artificial intelligence (AI) built into it. In 1956 John McCarthy proposed that the study of artificial intelligence be based on the fact that every aspect of learning can in principle be so precisely described that a machine can be made to simulate it [6]. Popenici and Kerr [7] propose a basic definition of AI as computing systems that are able to engage in human-like processes such as learning, adapting, synthesizing, self-correction and use of data for complex processing tasks. One prospective area of AI is machine learning (ML) where the computer learns from experience how to behave in new situations that are not explicitly instructed by the programmer or built into the software. This could involve tasks like weather prediction; face recognition or student categorization into closely related persona. A recent instance of ML is the AlphaGo developed by DeepMind at Google which defeated the human world champion at Go, a complex board game of strategy [8].

According to [9] the application of artificial intelligence in educational contexts may provide insights into how learning and teaching take place and possibly transform the way knowledge is evaluated in the system. It may also have a role in reorganizing classrooms or completely outdating them; the process can also enhance teaching efficiency or compel students to consider the role of human agency in probable outcomes. The $21 \mathrm{st}$ century is possibly a good time to initiate the thinking process as to what artificial intelligence could indicate for teaching, education and learning. The arrival of AI in our everyday life has made it almost impossible to avoid the pressing debate concerning its future role in teaching and learning. The outcome of this sensitive debate is ultimately set to determine what kinds of choices our present and future universities will have at their disposal with regard to higher education. It is becoming more and more evident that the future of higher education is intimately connected to the developments of new intelligent technologies and powerful computing capacities of the new millennium.

Kandlhofer et al. [10] argue that AI will form a very important part of education in the future, right from kindergarten level up to the universities, where possible content could include approaches to learning agents (tools that are capable of learning from experience) such as logic-based learning, knowledge-based systems or reinforcement learning, as well as decision trees and neural networks. Advances in AI have opened a host of new possibilities and at the same time revealed serious challenges for learning and teaching in modern higher education. The shift of the mode of teaching to a technology-dependent paradigm can potentially change the internal architecture of educational institutions or fundamentally transfer their governance from a body of able- minded persons to a system of interconnected computers and teaching or administrative software tools hooked to cloud.

\section{STATE-OF-THE-ART OF AI IN EDUCATION}

The future of higher education in terms of artificial intelligence is of substantial consequence. There are new technologies developing daily which integrate assistance from advanced, competent machines. In this field, progressions in artificial intelligence provide endless possibilities and consequences for learning and teaching in higher education, with the capacity to essentially alter governance and infrastructures of institutions. Education is an intricate and dynamic process, which comprises different goals and objectives. 
AI is clearly progressing with giant steps, perturbing facets of higher education on an unprecedented scale, not only transforming the way in which students learn, but also remodeling the entire architecture and landscape of education, particularly the universities.

\subsection{IBM Watson supercomputer}

One example of artificial intelligence deployed in a higher education institution is the supercomputer Watson from IBM. Watson is an IBM supercomputer that combines artificial intelligence (AI) and sophisticated analytical software for optimal performance as a "question answering" machine. The supercomputer is named for IBM's founder, Thomas J. Watson, and it is currently advising students at an Australian University whenever any help is needed, at any time, accessible from any place, on topics ranging from car parking to submission of assignments [11]. Watson is reported to work on a wide range of different machine learning algorithms where both shallow and deep knowledge like neural network (NN) are integrated by balancing the use of strict and shallow semantics. Interestingly, none of the individual components give the final answer, rather each of the components suggest features and their level of confidence, while an underlying sub- layer learns how to compile and combine them. While the nature of Watson's services may be argued to be more comprehensive and derivative rather than innovative or revolutionary, its impact on the quality of services, and the inevitable reduction in the demands on the administrative workforce is already amply evident. Interestingly, as universities strive to find viable solutions to fight their reduced budgets, AI comes in as a ready solution in hand.

\subsection{Brain-computer interface (BCI)}

Another facet of this automation of education involves the brain-computer interface (BCI). BCI is a collaboration between a brain and a device that enables signals from the brain to direct some external activity, such as control of a cursor or a prosthetic limb. The interface enables a direct communications pathway between the brain and the object to be controlled [12]. BCI is working hand in hand with artificial intelligence, which seems to open grand new possibilities toward rethinking (or perhaps even replacing) the role of the teacher in the learning process. Steps toward replacing teachers with teacher-bots is no more a fiction, but a crossroad where we are standing at present $[13,14]$. As an example, a BCI device is capable of measuring the extent to which a student is following or staying focused on the learning tasks provided [15, 16]. Gonzales et al. [16] have used ANOVA to analyze EEG data obtained directly from subjects' brains through NeuroSky MindWave devices attached to their heads. It is reported that the concentration patterns of creative, debugging and systematic programming tasks are quite different in time and intensity. Ehsan et al. [17] used an encoder-decoder network to generate relevant natural language explanations for any given action and locate them along the dimensions of confidence, human likeness, adequate justification, and understandability. The encoder and decoder are both recurrent neural networks $(\mathrm{RNN})$ comprised of gated recurrent unit (GRU) cells.

\subsection{Teacher-robots}

A more advanced use case was reported from a US University, where a teaching assistant (TA) was about to be nominated for the outstanding TA award. This was an evident reflection of how the TA best met the expectations and secured the highest evaluation from students. To everyone's surprise, the TA Jill Watson is a teacher-bot, the virtual teaching assistant built on the IBM Watson platform [18]. Jill Watson 1 was developed using the IBM Blue mix tool suite, using an episodic memory of questions and their answers from previous episodes. In the second and third generations, the design of Jill Watson $2 \& 3$ steadily moved from using an episodic memory of previously successful question-answer pairs to using semantic processing based on conceptual representations. A teacherbot is essentially a complex algorithm, which acts as an interface to provide personalized education through artificial intelligence. It supplies highly customized, adaptive educational content to students and provides appropriate supervision and guidance to teachers as well as students and scholars. Teacher-bots may be defined as machine-based software or hardware that assumes the role traditionally performed by a teaching assistant in organizing information and providing fast answers to a wide set of predictable questions [7].

\subsection{Virtual learning companion (VLC)}

Coming back to Turing's definition of intelligence, a virtual learning companion (VLC) such as an automated but intelligent teaching assistant needs to be able to simulate human-like behavior so that its inter- actions with students appear realistic and believable. A Curious Companion proposed by Wu et al.[19] aims to provide personalized content to students in a virtual learning milieu. The authors adopted the matrix factorization (MF) in a class of collaborative filtering algorithms used in recommender systems. Matrix Factorization algorithms work by decomposing the user-item interaction matrix into the product of two 
lower dimensionality rectangular matrices. MF algorithms have been shown to perform well for recommender systems, especially when the data is sparse. It is a method for measuring user preferences, wherein experimental results reveal that the incorporation of social curiosity information significantly improves the precision, coverage, and diversity of recommender systems. By noting and analyzing every student's progress, the intelligence of the companion lies in finding out which learning materials would arouse curiosity in the student's mind, maintain interest and flow in learning, and avoid distraction.

A similar 'remembrance' companion [20] could help learners manage a huge volume of information and knowledge. The data is first stored in the episodic memory, and then gets a more consolidated form in the semantic memory of the companion akin to ways in which humans organize and retrieve information in their memory. When a student is stuck on a question, the companion provides the most relevant episodes in the episodic memory or nearest concepts in its semantic memory to extend help in answering the question. The intelligent agent is modelled as a multi-class classification problem for episodes, with each concept representing a class label. Possible over- dependence of a student on the companion is addressed through progressive interaction with a series of more and more explicit hints until the student is able to recall the exact information required. Another learning companion, Bettys Brain, helps students to integrate their knowledge [21]. By using the learning-by-teaching approach, and through clustering similar students' behavior through coherence analysis into five distinct clusters, the Bettys Brain model has improved its ability to understand the different nature of difficulties that students face. Additionally, an open-ended learning environment helps prepare the students for future learning by developing and applying metacognitive strategies for setting goals and subsequently developing plans to achieve those goals. Carnell et al. [22] tried to predict the success of medical students in interviewing virtual patients to know their diet and eating habits or history of present illness through different machine learning algorithms such as: Naive Bayes, k-Nearest Neighbors, Logistic regression, support vector machine (SVM), CART, and Bayesian Rule Lists. In a similar study [23] Mathematics and Business Management students in a University were studied, and the final score of students were predicted before participating in the final examination using a neural network (NN) modeled with radial basis functions. Quite a few students identified in the weak zone, and suggestions proposed for improvement. Acknowledged theories of education are utilized by VLCs by implementing learning through teaching or argumentative learning, where VLCs can help learners by organizing the accumulated information in the form of coherent knowledge through fruitful discussions or effective summarizations [24]. Modelling a learning path for course introductions (e.g. the Human-Computer Interaction course, where the syllabus is not fully defined, or where individuals may be perceived to have different learning paths) has been constructed using the software Goal Net, which was created and copyrighted by the authors [24].

\subsection{Massive open online course (MOOC)}

With the potential to reach a huge section of learners across the globe, MOOCs have become an important tool in higher education today. The leader in online teaching, edX defines a MOOC at their extended website mooc.org as free online courses available for anyone to pursue, providing an affordable and flexible way to learn new skills and advance one's career through quality educational experiences at scale [25]. Although MOOC is not an AI technology, it is a platform where AI may be applied In a MOOC, predictive models of student behavior can support multiple aspects of learning, including instructor feedback and timely intervention. MOOCs currently follow classroom-teaching patterns, from the delivery of course content to selecting exercises, conducting tests and evaluating progress, with pre-determined components for an anonymous student to fit in mechanistically. A criticism of this method is that it leaves little scope for individuality, creativity or critical thinking [24]. Another allegation directed against MOOCs is their inability to provide sufficient hands-on exercises to help learners develop practical skills from the taught theories and concepts [26]. Another study reports high dropout rates in case of many MOOCs [27], where three different kinds of predictive analysis were performed to study the dynamics of dropouts.

Adopting new technologies for higher education needs to be explored, and the future of a world where $\mathrm{AI}$ is part of the fabric of universities must be predicted. Clearly, it is challenging to adopt these technologies for teaching, learning, student support, and administration. However, their capability in measuring the extent to which a student is following or staying focused has clear potential to benefit both students and teachers in understanding each other either, in better relating the lessons, and by improving the assessment of students in higher education courses. Unable to answer 10,000 questions from students each semester, Ashok Goel planned to employ the virtual TA to be able to answer nearly half of all questions asked by students in a medical education course. The students interviewed several virtual patients about their diet and eating habits and history of present illness, and the success of the students in the interview was predicted with the help of several ML algorithms. The MOOC platform provided support for personalizing of learning, richer interaction opportunities and analytics. Firstly, a linear regression analysis was used to gain an overview 
of how MOOC enrolments and completion rates were changing over time. Secondly, a multiple regression analysis was carried out to explore the combined effects of factors such as course length, start date and assessment type, on the completion rate of the courses following a Box-Cox transformation in each case to ensure that the residuals follow a normal distribution. Thirdly, a graphical visualization helped to reveal the week-by-week changes in participation of students in the courses either through attending later level programs or through submission rates of assignments. Noting the volume, variety and velocity of information generated by MOOCs, and acknowledging it as big data. Santur et al. [28] used a host of different algorithms (such as: linear regression for statistical tests, singular value decomposition for dimension reduction, k-means for clustering, random forests for classification and finally multi-layered neural networks for deep learning and building the model) to improve the content of MOOCs. The application of AI in MOOCs is not always straightforward. The main reason is likely to be that current MOOC teaching focuses on standardization rather than personalization. An interesting study on improving MOOC courses [24] proposes to analyze tailored learning paths and their respective outcomes vis-a'-vis individual students' backgrounds (data which MOOC platforms already possess) through machine learning. Such analyses could inform educational researchers, businesses, and policy makers on how to personalize the learning trajectory of students coming from different ethnic or educational backgrounds in the cases of particular courses. Table 1 summarizes the most important works reviewed in this section.

Table 1 . Summary of the important works reviewed

\begin{tabular}{|c|c|c|c|}
\hline Ref & Year & Topic/Method & Features \\
\hline [7] & 2017 & $\begin{array}{l}\text { Extensive research on the past experiences and current } \\
\text { status of the application of AI in higher education }\end{array}$ & $\begin{array}{l}\text { Adopting new technologies for higher education } \\
\text { are explored; future of a world where AI is part } \\
\text { of the fabric of universities is predicted. Some } \\
\text { challenges in adoption of these technologies for } \\
\text { teaching, learning, student support, and administration } \\
\text { is pointed out. }\end{array}$ \\
\hline [16] & 2015 & $\begin{array}{l}\text { Brain-Computer Interface (BCI) devise, NeuroSky's } \\
\text { Mind wave, employing EEG signals from human brain }\end{array}$ & $\begin{array}{l}\text { Capability of measuring the extent to which a student } \\
\text { is following, or staying focused on the learning tasks } \\
\text { he/she is provided }\end{array}$ \\
\hline [17] & 2019 & $\begin{array}{l}\text { An encoder-decoder network was developed using } \\
\text { Recurrent Neural Networks (RNN) based on Gated } \\
\text { Recurrent Units (GRU) to explain moves in a way } \\
\text { understandable to humans }\end{array}$ & $\begin{array}{l}\text { The explicability of narrations generated automatically } \\
\text { through this application can benefit both student and } \\
\text { teachers in understanding each other either in better } \\
\text { relating the lessons, or by improving the assessment } \\
\text { of students in higher education courses. }\end{array}$ \\
\hline [22] & 2019 & $\begin{array}{l}\text { Several classifiers, viz. Naive Bayes, k-NN, SVM, CART } \\
\text { and Bayesian Rule Lists utilized to assess students on } \\
\text { their interviews of virtual patients in a medical course }\end{array}$ & $\begin{array}{l}\text { The students interviewed several virtual patients about } \\
\text { their diet and eating habits and history of present } \\
\text { illness, where the success of the students in } \\
\text { the interview was predicted with the help of several } \\
\text { ML algorithms }\end{array}$ \\
\hline [24] & 2017 & $\begin{array}{l}\text { Offers perspective on how advances in AI may enhance } \\
\text { learning and research on MOOCs; assistants to offer } \\
\text { personal attention to learners }\end{array}$ & $\begin{array}{l}\text { MOOC platform supports for personalizing of learning, } \\
\text { richer interaction opportunities and analytics }\end{array}$ \\
\hline
\end{tabular}

\section{EFFECTIVE COURSE ASSESSMENT}

Effective course assessment can be defined as the techniques, strategies, instruments, and tools for the collection of knowledge and information to measure the extent to which students represent and project required learning outcomes. This is possibly the most effectual and integrated aspect of online and offline education. Course assessment is significant because it is known to have a supportive influence on learning and provide a sense of the quality of learning taking place in a classroom setting. According to the explanations provided by [29], course assessment is the sequential collection and analysis of data for the improvement of student learning and insight development. This definition provides a clear projection of the essential functionality of student assessment in the learning and teaching process. Student assessments and evaluations allow instructors to evaluate and quantify the efficacy of their teaching along with the style of course management and regulation by associating student performance to particular learning objectives. As an outcome, teachers would be capable of institutionalizing effectual teaching mechanisms and revising failed ones in their domains. 
Comparing the outcomes and discussions of [30] and [31], it should be mentioned that the course assessment process is beneficial and effective. The process of course assessment for the measurement and assessment of student learning is important because it delivers significant feedback to both teachers and students about the scope and magnitude to which students are consistently fulfilling learning objectives of the course. In their book Understanding by Design, Wiggins and McTighe [31] provide a framework for classroom management and knowledge development by emphasizing the fundamental function of assessment; the term "backward design" has been used in an interrelated manner. According to the authors, course assessment al- lows teachers to regulate the metrics of measurement for student understanding and of aptitude, expertise and developmental skills in course learning objectives. They discuss that an effectual assessment takes into consideration the evidence required to outline and confirm that substantive learning has followed in the classroom [[31]. Course assessment is so critical in their instructional design that their strategy has inspired tutors and curriculum planners to initially reflect like an appraiser before establishing particular lessons and units, and therefore to consider directly how they will determine if the students have understood the concepts or are essentially capable of delivering similar theoretical concepts.

\subsection{Process of effective course assessment}

The process of effective course assessment involves different methods of evaluation consider by Brown and Knight [32]; these can be applied in the classroom with consistency and regularity. It is important to take into consideration that these techniques work effectively when learning objectives have been highlighted in a constructive manner along with suitable sharing and clear articulation to the students. An effective course assessment includes self-assessment, peer assessment, time-constrained and examination-based individual assessment. Self-assessment is defined as the process of implementing different guidelines and approaches for students through which they can measure their own capabilities and academic attributes. For self-assessment, students are estimated to test both procedures and outcomes of their learning. While the final evaluation is often performed by the teacher, implementing student assessment in the learning environment motivates students to excel and test their own educational tendencies along with the practices that resulted in the final outcome [32]. This kind of assessment leads students to initiate transferable aptitudes into additional learning areas that incorporate teamwork and group tasks, problem-solving, and critical rationalization. Various self-assessment $\operatorname{logs}$ are produced and cultivated from the data and information of artificial intelligence, which can lead to successful self-assessment in higher education [32].

Peer assessment is a form of collaborative assessment where students check the work of other students and have their own functionalities evaluated as well [32]. This aspect of assessment is considerably articulated in theoretical approaches for adult learning and active learning. Both self-assessment and peer assessment provide a sense of ownership and accountability in learners. Examinations have generally been perceived as a remarkable assessment standard in education, specifically in higher education settings such as colleges and universities [32]. These may involve either formative or summative forms of evaluations depending on the nature of the course and the learning objectives.

\subsection{Efficient course assessment}

The underscored purpose of this and the following literature survey sections is to find an alternative assessment procedure, its impact on classroom instructions, modifications to curriculum planning, student learning and to obtain or to give input to the higher education teachers perception and assess with as little bias as possible the implications of various assessment procedures suggested in those models. Brink and Bartz [33] suggest the change of perception of high school teachers toward formative assessment, while Landry et al. [34] propose the effective use of peer assessment in the form of comments and feedback in graduate writing assessments. Ahankari and Jadhav [35] placed confidence in e-rubrics in an effort to align formative as well as summative assessment tools for assessment of course and program outcomes. Neoman et al. [36] have tried to evolve a quality assessment model toward achieving quality standards. A model proposed by Kerdijk et al. studies the effects on the students of self-study time and test performance by contrasting cumulative versus end-of-the-course assessments [37]. A tool for outcomes assessment, student skill and course improvement through pre- and post-course student self-assessment was studied by Jamieson and Shaw [38]. Mandernach [39] has offered a useful synthesis of literature regarding assessment tools and the assessment of student engagement in higher education in a paper. In the case of information technology courses, a glimpse of students' assessment regarding online versus face to face delivery has been studied by Said et al.[40]. Sustainable assessment is reviewed in yet another work [41]. A few recent works deal with AI-powered personalization [24], AI-based assessment systems [42], automatic discovery of skill models for online courses [43], and students' performance with Machine Learning methods [44]. 


\subsection{Preparing the teachers towards formative assessment}

For the preparation of higher education teachers, content knowledge, rather than instructional methodologies, is the greater focus [45]. In this context, formative assessment becomes an important tool to facilitate two-way communication between the students and the teacher. Encouraging such modifications of teachers' practices would enable the students to self-assess and utilize available tools to improve their own learning. Popham [46] discusses statistical concepts of reliability, bias in teaching and ways to interpret standardized test scores such as percentiles, using a layman's approach that enables teachers to grasp the meaning and significance of these mathematical terminologies. Hosp and Ardoin [47] stress the detailed procedures for correctly measuring accuracy or fluency of performance, and for choosing appropriate measures to provide correct and relevant information about the students to instructors. A comprehensive staff development program is also essential and must be personalized to the needs of each teacher, and furnish examples of state-of-the-art use of formative assessment on a department by department basis. This means that the institutional administrations should embrace the culture of formative assessment as standard operating procedure in all classrooms [33]. Suresh et al. [48] have developed an innovative application called 'TalkBack' to provide a source of feedback to teachers based on the automated analysis of classroom recordings. The application builds on advances in deep learning for natural language processing and speech recognition. It automatically analyzes classroom recordings, including an embedded and automated "talk move" classifier. By providing teachers with a detailed record of the discourse strategies used in their lessons, the application can dramatically enhance teacher learning and support improvements in their instruction.

\subsection{Automating the assessment process using AI}

The use of automated assessments can offer detailed and immediate feedback to students related to their learning progress and can help in identifying the areas in which the students need to improve their efforts. Hence, MOOC related courses have been using deployed automated assessments for objective testing. The types of multiple-choice questions, which these assessments employ, tend to be highly reliable when they are graded and administered by computer [49]. These types of learning environments tend to permit the instructors to improve their use of multiple choice assessments and enrich the learning experience for their students. There are also automated essay scoring AI tools, which can be used for subjective evaluations. These kinds of tools can result in reduced time and costs related to human efforts of rating, interpreting and reading students' work. This process starts with developing a set of training essays, which are scored by the instructor. Such a process was designed for the MOOC courses offered by EdX, which required the instructor to manually score 100 essays to ensure that the machine algorithms of could learn to score and give feedback as well as a human instructor [49].

Hence, based on the use of scoring examples, using the scored examples, the application can learn to evaluate essays in terms of frequency of work, use of vocabulary, average length of word and grammar. This shows that based on the training set, the AI develops a statistical model that predicts the scores, which were human, assigned. There are also more advanced systems, which deploy natural language process techniques such as semantic analysis, sentiment analysis and summarization of text[48]. In this regard, edX, which is a nonprofit online course provider cofounded by MIT has developed an automated system of essay scoring called the Enhanced AI Scoring Engine [50]. Through this system, edX has been making the machine graders more human-like, because instead of only scoring the essays based on a specific rubric, the software tends to mimic grading styles of specific professors. This happens because every professor tends to score essays based on their own criteria. Thus, the system picks up those patterns and assimilates them into their machine marking. The main aim is to develop an automated version of a professor, which can give feedback on large number of essays, resulting in improved formative assessments done at a higher speed [50]. Ideally, a course will use a combination of different methods so that each method can do a check on the other. In case human graders and the system assign different scores, the instructor might be called to giving an expert opinion [50].

Along with this automated scoring of essays, calibrated peer review is another important application, which focuses on managing the workflow of the peer review process and tends to score on how well the peer reviews are performed. This system enables a large number of students to submit their essays, learn about the critical points that are highlighted by the instructor through a multiple-choice rubric, do a peer review of the work of their fellow students, self-evaluate their own work and receive feedback from the peers who have reviewed their work. In terms of the effectiveness of this system, studies have revealed that calibrated peer review (CPR) enables students to learn the content, which they have written about as well as improve their specific skills related to writing [51]. 


\subsection{Student's assessment through online course vis-a' -vis face to face delivery}

Online programs continue to experience growth in enrolment in response to the continued penetration of communication and information technologies into all sectors of society and the growing needs for qualified professionals to help users and organizations utilize these technologies. Yet it should be noted that several studies reviewed by Weber and Lenon [52] showed mixed results in students' performance and satisfaction across different courses and disciplines. While some studies found online courses to be superior, and others found face-to-face courses to be superior, the majority of studies found no difference in learning outcomes or students' satisfaction between the two course delivery modes [40]. Analysis of various data indicates that in the overall rating of course quality and instructor performance, course organization has received the highest ratings in online courses while the instructor's performance is perceived to be superior in face-to-face courses. Additional analysis shows that students have ranked instructor performance as the most important academic success factor [40]. Said et al. [40] analyzed the data with the SPSS software, using a split plot factorial ANOVA, which provided useful insights to the existence and degree of interaction between the course quality indicators and the course structure. One across-the-group factor was chosen to represent the course structure (on-line delivery or face-to-face delivery) and another across-the-group factor was used for representing the course quality indicators. However, in some online courses, the role of the instructor might not be as valuable, since in courses where students are focused on applying their knowledge to case studies, students in online sections act more independently and may not be as reliant on the instructor as they would be in face-to-face courses [40]. However, Bergstrand and Savage [53] found that students felt that they received less respect from the instructors and that enthusiasm, preparedness and skills were important factors even in online courses. The authors [53] used the hierarchical linear modeling (HLM) to analyze the data, as this allows evaluation of data that are nested in larger units such as organizations, universities or countries. Inheriting the ability to incorporate independent variables at both the individual and cluster levels, their methodology produced a multilevel model to assess the effects of course-level variables, as well as instructor-level variables on evaluation. They used a full maximum likelihood estimation, conducting the analyses through the Stata software.

What is important is how to make all courses a meaningful experience for students, regardless of the mode of instruction. There is room for improvement in delivery methods in both kinds of courses. Instructors for online courses need to extend their availability and improve their communication with students. Academic departments can urge their instructors to obtain training on the use of activities and tools that increase the level of one-to-one and group communication between instructors and students [40]. In face-to-face courses, the instructor's presence in the classroom can sometimes reduce the priority given to other course quality factors such as organizing the course content, pacing the learning and assessment activities, providing prompt written feedback to each student, and clarifying the learning outcomes throughout the semester [40].

In spite of the deficiencies in the automatic question answering system of education, the traditional face-to-face approach to answering questions, both for educators and learners, has been noted as grossly insufficient in meeting student needs [54]. A mixed deep neural network is constructed based on the long short-term memory (LSTM) or gated recurrent unit (GRU), in order to learn the deeper characteristics of a sentence better through deep learning and word2vec approaches.

\subsection{Use of $A I$ in sustainable assessment of course}

As far as we search in our discussions, we find that whatever sustainability in assessment of courses has been reached, it is very much reliant on personalized practices of the instructors and the students be it the outcome of changed institutional organization or be it the upgrading of online course indicators. For example, Huang et al. [55] have used deep neural networks (DNNs) to develop a model to predict whether the student would answer each exercise in a video lesson correctly, and was thereby able to recommend videos that suited the level of the student, and that he/she would be able to follow the best.

\section{CONCLUSION}

This paper is a review of the state of the art with respect to artificial intelligence. It has surveyed the most relevant artificially intelligent applications in education, especially in course assessment. The progression that is taking place in the field of artificial intelligence tends to offer endless possibilities as well as consequences for the purpose of learning and teaching in education. This explosion of AI applications in education is epitomized through many applications such as Jill Watson (a teaching assistant who successfully answers questions coming from students), MOOCs (modern teaching innovations that provide personalized learning material for students from different backgrounds using machine learning algorithms), VLC, BCI, automatic essay scoring and other applications that have been mentioned earlier. In this regard, 
studies have highlighted that with the progress and advancement in the field of AI, it is expected that the gaps and inefficiencies in education will ultimately be filled, enabling the educational systems to accomplish more than what has been achieved to date. It can perhaps be concluded that while no technology has completely replaced the human touch in teaching so far, artificially intelligent applications have reached far enough in their ability to carry the essence of existing teaching practices by molding and re-forming them in a more acceptable and friendly way. This is primarily due to the scalable and powerful features of the new technology, which make it adaptable to different situations where every individual belongs.

\section{REFERENCES}

[1] Artificial Intelligence (AI): What Is It and How Does It Work? | Lexology. [Online]. Available: https://www.lexology.com/library/detail.aspx?g=5424a424-c590-45f0-9e2a-ab05daff032d.

[2] M. A. Boden, "AI: Its Nature and Future,” Oxford University Press, 2016.

[3] Müller V.C., Bostrom N., "Future Progress in Artificial Intelligence: A Survey of Expert Opinion," Fundamental Issues of Artificial Intelligence, vol. 376, pp. 555-572, 2016.

[4] S. Armstrong, N. Bostrom, and C. Shulman, "Racing to the Precipice: A Model of Artificial Intelligence Development," AI \& Society, vol. 31, no. 2, pp. 201-206, 2016.

[5] Artificial Intelligence. [Online]. Available: https://en.wikipedia.org/wiki/Artificial intelligence.

[6] S. J. Russell and P. Norvig, "Artificial Intelligence: A Modern Approach,” Pearson Hall, Malaysia, 2016.

[7] S. A. Popenici and S. Kerr, "Exploring the Impact of Artificial Intelligence on Teaching and Learning in Higher Education," Research and Practice in Technology Enhanced Learning, vol. 12, no. 22, 2017.

[8] E. Gibney, "Google Reveals Secret Test of AI Bot to Beat Top Go Players," Nature News, vol. 541, no. 7636, 2017.

[9] D. Acemog lu and P. Restrepo, "The Race Between Machines and Humans: Implications for Growth, Factor Shares and Jobs," American Economic Review, vol. 108, no. 6, pp. 1488-1542, 2018.

[10] M. Kandlhofer, G. Steinbauer, S. Hirschmugl-Gaisch, and P. Huber, "Artificial Intelligence and Computer Science in Education: From Kindergarten to University,” 2016 IEEE Frontiers in Education Conference (FIE), pp. 1-9, 2016.

[11] Deakin IBM Watson. [Online]. Available: http://archive.li/kEnXm

[12] What is Brain-Computer Interface (BCI)? - Definition from whatis.com." [Online]. Available: https://whatis.techtarget.com/definition/brain-computer-interface-BCI

[13] S. Bayne, "Teacherbot: Interventions in Automated Teaching," Teaching in Higher Education, vol. 20, no. 4, pp. 455-467, 2015.

[14] L. Botrel, E. Holz, and A. Kübler, "Brain Painting V2: Evaluation of P300-Based Brain-Computer Interface for Creative Expression by An End-User Following the User-Centered Design," Brain-Computer Interfaces, vol. 2, no. 2-3, pp. 135-149, 2015.

[15] X. Chen, Y. Wang, M. Nakanishi, X. Gao, T.-P. Jung, and S. Gao, "High-Speed Spelling with A Noninvasive BrainComputer Interface," Proceedings of the national academy of sciencesof the United States of America, vol. 112, no. 44, pp. E6058-E6067, 2015.

[16] V. M. Gonza'lez, R. Robbes, G. Go'ngora, and S. Medina, "Measuring Concentration While Programming with Low-Cost BCI Devices: Differences Between Debugging and Creativity Tasks," International Confer-ence on Augmented Cognition, vol. 9183, pp. 605-615, 2015.

[17] U. Ehsan, P. Tambwekar, L. Chan, B. Harrison, and M. Riedl, "Automated Rationale Generation: A Tech- Nique for Explainable AI and Its Effects on Human Perceptions," 2019 International Conference on Intelligent User Interfaces, 2019.

[18] J. Maderer, "Artificial Intelligence Course Creates AI Teaching Assistant," Georgia Tech News Center, 2016.

[19] Q. Wu, S. Liu, C. Miao, Y. Liu and C. Leung, "A Social Curiosity Inspired Recommendation Model to Improve Precision, Coverage and Diversity," 2016 IEEE/WIC/ACM International Conference on Web Intelligence (WI), pp. 240-247, 2016.

[20] Q. Wu, X. Han, H. Yu, Z. Shen, and C. Miao, "The Innovative Application of Learning Companions in Virtual Singapura," Proceedings of the 2013 International Conference on Autonomous Agents and Multi Agent Systems, pp. 1171-1172, 2013.

[21] G. Biswas, J. R. Segedy, and K. Bunchongchit, "From Design to Implementation to Practice A Learning by Teaching System: Betty's Brain," International Journal of Artificial Intelligence in Education, vol. 26, no. 1, pp. 350-364, 2016.

[22] S. Carnell, B. Lok, M. T. James, and J. K. Su, "Predicting Student Success in Communication Skills Learning Scenarios with Virtual Humans," Proceedings of the 9th International Conference on Learning Analytics \& Knowledge, pp. 436-440, 2019.

[23] M. Ciolacu, A. F. Tehrani, L. Binder and P. M. Svasta, "Education 4.0 - Artificial Intelligence Assisted Higher Education: Early recognition System with Machine Learning to support Students' Success," 2018 IEEE 24th International Symposium for Design and Technology in Electronic Packaging (SIITME), pp. 23-30, 2018.

[24] H. Yu, C. Miao, C. Leung, and T. J. White, "Towards AI-Powered Personalization in MOOC Learning," npj Science of Learning, vol. 2, no. 15, 2017.

[25] MOOC.org. [Online]. Available: http://mooc.org/

[26] M. M. Waldrop, “Education Online: The Virtual Lab”, Nature News, vol. 499, no. 7458, pp. 268-270, 2013.

[27] K. Jordan, "Massive Open Online Course Completion Rates Revisited: Assessment, Length and Attrition," 
The International Review of Research in Open and Distributed Learning, vol. 16, no. 3, 2015.

[28] Y.Santur, M. Karaköse and E. Akin, "Improving of Personal Educational Content Using Big Data Approach for MOOC in Higher Education," 2016 15th International Conference on Information Technology Based Higher Education and Training (ITHET), pp. 1-4, 2016.

[29] M. L. Stassen, K. Doherty, and M. Poe, "Course-Based Review and Assessment: Methods for Understanding Student Learning. Office of Academic Planning \& Assessment," Robert Langhorst \& Company Booksellers, Amerika Serikat, 2001.

[30] A. W. Astin, T. W. Banta, K. P. Cross, E. El-Khawas, P. T. Ewell, P. Hutchings, T. Marchese, K. McClenney, M. Mentkowski, M. Miller et al., "Principles of Good Practice for Assessing Student Learning," Leadership Abstracts, vol. 6, no. 4, 1993.

[31] G. Wiggins and J. McTighe, "Understanding by Design," Alexandria, US, 2005

[32] S. Brown and P. Knight, "Assessing Learners in Higher Education," Routledge, 2012.

[33] M. Brink and D. E. Bartz, "Effective Use of Formative Assessment by High School Teachers," Practical Assessment, Research \& Evaluation, vol. 22, no. 8, 2017.

[34] A. Landry, S. Jacobs, and G. Newton, "Effective Use of Peer Assessment in A Graduate Level Writing Assignment: A Case Study." International Journal of Higher Education, vol. 4, no. 1, pp. 38-51, 2015.

[35] S. S. Ahankari and A. A. Jadhav, "e-Rubrics: A Formative as Well as Summative Assessment Tool for Assessment of Course and Program Outcomes," 2016 IEEE Eighth International Conference on Technology for Education (T4E), pp. 246-247, 2016.

[36] A. Y. Noaman, A. H. M. Ragab, A. I. Madbouly, A. M. Khedra, and A. G. Fayoumi, "Higher Education Quality Assessment Model: Towards Achieving Educational Quality Standard," Journal Studies in Higher Education, vol. 42, no. 1, pp. 23-46, 2017.

[37] W. Kerdijk, J. Cohen-Schotanus, B. F. Mulder, F. L. Muntinghe, and R. A. Tio, "Cumulative Versus End- of-Course Assessment: Effects on Self-Study Time and Test Performance,” Medical Education, vol. 49, no. 7, pp. 709-716, 2015.

[38] M. V. Jamieson and J. M. Shaw, "Pre and Post Course Student Self-Assessment of Ceab Graduate Attributes- A Tool for Outcomes Assessment, Student Skill and Course Improvement," Proceedings of the Canadian Engineering Education Association (CEEA), 2016.

[39] B. J. Mandernach, "Assessment of Student Engagement in Higher Education: A Synthesis of Literature and Assessment Tools," International Journal of Learning, Teaching and Educational Research, vol. 12, no. 2, pp. 1-14, 2015.

[40] H. Said, L. Kirgis, B. Verkamp, and L. Johnson, "On-Line vs. Face-to-Face Delivery of Information Technology Courses: Students' Assessment,” Journal of Information Technology Education: Research, vol. 14, pp. 297-312, 2015.

[41] D. Boud and R. Soler, "Sustainable assessment revisited," Journal Assessment \& Evaluation in Higher Education, vol. 41, no. 3, pp. 400-413, 2016.

[42] R. Luckin, "Towards Artificial Intelligence-Based Assessment Systems," Nature Human Behaviour, vol. 1, no. $0028,2017$.

[43] N. Matsuda, T. Furukawa, N. Bier, and C. Faloutsos, "Machine Beats Experts: Automatic Discovery of Skill Models for Data-Driven Online Course Refinement." International Educational Data Mining Society, Paper presented at the International Conference on Educational Data Mining (EDM), 2015.

[44] M. Ciolacu, A. F. Tehrani, R. Beer and H. Popp, "Education 4.0 - Fostering Student's Performance with Machine Learning Methods," 2017 IEEE 23rd International Symposium for Design and Technology in Electronic Packaging (SIITME), pp. 438-443, 2017.

[45] A. F. Wong, S. N. Y. Chong, D. Choy, I. Y.F. Wong, and K. C. Goh, "A Comparison of Perceptions of Knowledge and Skills Held by Primary and Secondary Teachers: from the Entry to Exit of Their Preservice Programme," Australian Journal of Teacher Education, vol. 33, no. 3, pp. 77-93, 2008.

[46] W. J. Popham, "Classroom Assessment: What Teachers Need to Know (7th Edition), 2014.

[47] J. L. Hosp and S. P. Ardoin, “Assessment for Instructional Planning," Assessment for Effective Intervention, vol. 33, no. 2, pp. 69-77, 2008.

[48] A. Suresh, T. Sumner, I. Huang, J. Jacobs, B. Foland and W. Ward, "Using deep learning to automatically detect talk moves in teachers'mathematics lessons," 2018 IEEE International Conference on Big Data (Big Data), pp. 5445-5447, 2018.

[49] P. J. van Vliet, "Scaling Up Student Assessment: Issues and Solutions," Journal of Higher Education Theory and Practice, vol. 16, no. 6, pp. 32-47, 2016.

[50] S. Kolowich, "Writing Instructor, Skeptical of Automated Grading, Pits Machine vs. Machine," The Chronicle of Higher Education, vol. 28, 2014.

[51] S. P. Balfour, "Assessing Writing in MOOCs: Automated Essay Scoring and Calibrated Peer Review ${ }^{\mathrm{TM}}$," Research \& Practice in Assessment, vol. 8, pp. 40-48, 2013.

[52] J. M. Weber and R. Lennon, "Multi-Course Comparison of Traditional versus Web-Based Course Delivery Systems." Journal of educators online, vol. 4, no. 2, p. n2, 2007.

[53] K. Bergstrand and S. V. Savage, "The Chalkboard Versus the Avatar: Comparing the Effectiveness of Online and In-Class Courses," Teaching Sociology, vol. 41, no. 3, pp. 294-306, 2013.

[54] L. Wang and W. Wang, "Research and Construction of Junior High School Subject Q\&A System Model based on Deep Learning," 2018 International Conference on Information Systems and Computer Aided Education (ICISCAE), pp. 504-508, 2018.

[55] N. Huang, C. Chen, J. Tzeng, T. T. Fang, and C. Lee, "Concept Assessment System Integrated with A Knowledge Map Using Deep Learning,”2018 Learning with MOOCS (LWMOOCS), pp. 113-116, 2018. 